\title{
The Impact of Transition Training on Adapting to Technically Advanced Aircraft at Regional Airlines: Perceptions of Pilots in Training and Instructor Pilots
}

\author{
John C. Di Renzo Jr. \\ Joint Interagency Task Force-South, US Southern Command
}

Timm J. Bliss

Oklahoma State University

\begin{abstract}
The purpose of this research study was to test a hypothesis regarding newly trained regional airline pilot and instructor pilot (IP) perceptions of how effectively pilots learn and use new technology found in technically advanced aircraft (TAA), given initial type of instrumentation training. The research questions focused on the type of initial instrumentation training to determine the differences among pilots trained using various types of instrumentation ranging from aircraft equipped with traditional analog instrumentation to aircraft equipped with glass cockpits. This investigative study failed to disprove the null hypothesis $\left(\mathrm{H}_{0}\right)$ : The type of instrumentation training (during initial training) has no significant effect on newly trained regional airline pilots' perceived ability to adapt to advanced technology cockpits in more sophisticated and/or newer aircraft. Therefore, no evidence exists from this study to support the early introduction and training of TAA. While the results of this investigation were surprising, they are nonetheless instructive. Even though it would seem that there would be a relationship between exposure to and use of technically advanced instrumentation, apparently there was no perceived relationship for this group of newly trained airline transport pilots. However, the results of this investigative study raise many new questions and provide a number of ideas for future research projects.
\end{abstract}

\section{INTRODUCTION}

Attempts to instrument aircraft for communications, navigation, and for flight without reference to the natural horizon began in earnest in the 1920's (Bilstein, 2001). Since that time, great advances have been made in aviation related technologies. Where once a lack of aircraft systems was the limiting factor for flight into instrument meteorological conditions (IMC), now advanced aviation technologies found in technically advanced aircraft (TAA) present a significant challenge to pilots and crews trying to assimilate and effectively use ever increasing amounts of information.

The debate about how to best train future airline transport pilots abounds and new concepts regarding the role of active learning in scenario-based exercises are beginning to supplant more traditional approaches to flight training (FAA Education and Research, 2006). These new aviation technologies are pervasive and can be found in most aircraft, ranging from large commercial aircraft to small general aviation aircraft commonly used for initial flight training. Furthermore, the pace of technological advancement is increasing and will continue to present significant challenges for the aviation community (FAA Education and Research, 2006).

Given the proliferation of TAA, pilot training has become a point of critical interest. How should new pilots be trained and what is the effectiveness of that training as they move from the flight school to 
airline cockpits? Additionally, what is the role of traditional analog instrumentation training verses digital instrumentation training? Is training on analog instrumentation, digital instrumentation or a combination of both best for transition to TAA? Do pilots trained exclusively on traditional instruments experience more difficulty transitioning to TAA as compared to those pilots trained on a combination of analog and digital instrumentation, or as compared to those pilots exclusively trained on digital instrumentation?

The problem addressed in this study is to identify newly trained regional airline pilot and instructor pilot (IP) perceptions of their ability to learn and use advanced aviation technology. These systems are complex and a pilot must possess a significant degree of familiarity with automated systems. Much needs to be known about how current training of new pilots/crews prepares them to adapt to technically advanced cockpits in more sophisticated commercial aircraft. The following hypothesis was established for this research study:

$\mathrm{H}_{0 \text { : }}$ The type of instrumentation training (during initial training) has no significant effect on newly trained regional airline pilot's perceived ability to adapt to advanced technology cockpits in more sophisticated and/or newer aircraft.

$\mathrm{H}_{1}$ : The type of instrumentation training (during initial training) has a significant effect on newly trained regional airline pilot's perceived ability to adapt to advanced technology cockpits in more sophisticated and/or newer aircraft.

\section{METHODOLOGY}

This study used a mixed method design to allow the researcher to make an interpretation whether the results from both sets of data (quantitative and qualitative) support or contradict each other. The quantitative component used a non-probability sample of pilots in training (PT) to gather data about student pilot perceptions regarding transition to technically advanced cockpits. The qualitative component used an open-ended opinion survey administered to the regional airline instructor pilots (IP) to determine their perceptions regarding the ability of newly trained regional airline pilots to learn and use advanced cockpit technology.

The quantitative component used an analysis of variance (ANOVA) statistical analysis design to compare the means of the independent groups (Fraenkel \& Wallen, 2003). The Shapiro-Wilk test was used to test for normality; to ensure the respondent sample came from a normally distributed population (Shapiro \& Wilk, 1965). The Levene test was used to determine homogeneity of variance. Additionally, the Levene test checked the assumption of the null hypothesis. That is, that the population variances were equal and no significant differences existed between the research groups (Levene, 1960).

This research study required newly trained regional airline pilots to make a self-assessment of ease of transition into TAA after completing advanced systems training with Pinnacle Airlines. Pilots were administered a questionnaire focusing on level of experience, initial training type and method, and overall level of perceived proficiency/ability at the completion of training. Type; therefore, indicated if PT received initial flight training on analog instrumentation, digital instrumentation or a combination of both. Method of training addressed whether pilots received traditional maneuver based training to the FAA Practical Test Standard (PTS), commonly referred to as "Stick and Rudder" training, or whether they received scenario based FAA Industry Training Standards (FITS) training. Note that either maneuver based training or scenarios based training may be conducted in either analog instrumented aircraft or 
digitally instrumented aircraft. PT were also asked about their perceived level of comfort flying TAA under varying circumstances - such as hand flying the aircraft to minimums in IMC or when piloting in an emergency situation. This investigation also sought to compare actual airline training data from the pilots in training to the initial training they received. However, access to proprietary training data was not available.

The study sought to answer the following four research questions:

1. What is the perceived ability of newly trained regional airline pilots initially trained on only analog systems to adapt to more advanced cockpit technologies?

2. What is the perceived ability of newly trained regional airline pilots initially trained on only digital systems to adapt to more advanced cockpit technologies?

3. What is the perceived ability of newly trained regional airline pilots initially trained on both analog and digital systems to adapt to more advanced cockpit technologies?

4. What are IP perceptions/observations of newly trained regional airline pilots' ability to adapt to TAA, as compared to the perceptions of the PT?

\section{Description of Research Instruments}

The first research instrument used in this study was a structured questionnaire created to ascertain PT attitudes regarding TAA training provided by Pinnacle Airlines. The questionnaire consisted of ten multiple-choice questions and 22 Likert scale questions. The multiple-choice questions were generated to collect demographic information and to determine the newly trained pilot's level of experience and comfort with TAA. Likert scale questions were used to determine pilot attitudes/perceptions about how initial training and proficiency with analog and/or digital systems affects ability to transition to advanced cockpits. For each Likert scale question, a score of five indicated strongly agree, four agree, three neutral, two disagree and one strongly disagree.

The study also incorporated a qualitative component to assess IP perceptions and opinions about how the pilots in training adapted to TAA training. A separate questionnaire of five open-ended questions was presented to the IP to compare their perceptions with those of the newly trained pilots. They were asked their opinions/observations across a number of subjects including, but not limited to:

- Benefits of scenario-based training (i.e. method of training)

- Maintenance of basic flying skills in a highly automated cockpit environment

- Benefits of the type of initial flight training received by pilots in training (i.e. analog only, digital only, or a combination of analog and digital training)

- Comfort level of PT flying TAA after completing training

To insure the highest level of quality, the research instruments were validated by testing with a small group of professional pilots and aviation industry professionals. There were changes made to the PT questionnaire because it was considered too lengthy and concerns were expressed that respondents would not fully complete the survey. There were no changes to the IP instrument.

\section{Selection of the Research Participants}

The PT sample was selected from pilots receiving initial training in technically advanced regional 
airline aircraft in 2008 at Pinnacle Airlines in Memphis, Tennessee. The PT sample included 46 male pilots and two female pilots. These 48 participants were categorized into three distinct groups: (Group One) pilots receiving analog only initial and instrument flight training using computer-based training (CBT), flight training devices (FTD), full motion simulators, and/or actual aircraft; (Group Two) pilots receiving both analog and digital initial and instrument flight training using CBT, FTD, full motion simulators, and/or actual aircraft; and (Group Three) pilots receiving digital only initial and instrument flight training using TAA cockpit CBT, FTD, full motion simulators, and/or actual TAA. After gathering and analyzing the research questionnaires, there was only one respondent in the digital only group. Consequently, this group was eliminated by the researcher from the statistical analysis section of the study.

Each class of Pinnacle students was trained for approximately six weeks at Pinnacle Airlines' primary training facility in Memphis, Tennessee. Pilots learned to fly the CRJ-200 TAA to FAA FAR Part 121 standards. The training included technology training on systems such as: Flight Management Systems (FMS), Electronic Flight Instrumentation Systems (EFIS), Engine Indication and Crew Alerting Systems (EICAS), Aircraft Systems and Operation, Crew Resource Management (CRM) and Emergency Procedures, Swept Wing Aerodynamics and Aircraft Performance, and the Pinnacle Airlines' Flight Operations Manual (FOM).

For purposes of this study, analog only training was conducted on traditional steam gauge instruments using the pitot static system and suction pump or electrically driven gyroscopic instrumentation. In addition, digital instrumentation training was conducted on CBT devices, FTDs and/or simulators that represented airspeed indicators, attitude indicators, altimeters, heading indicators, turn and bank coordinators and vertical speed indicators, and other instrumentation where these instruments were displayed on computer screens and powered by the electrical system (i.e. glass cockpit).

The second sample included four IP who provided training at Pinnacle Airlines during the time this study was conducted by the researcher.

\section{RESULTS}

The quantitative component of this study administered a closed-ended survey to 48 pilots in training, $46(96 \%)$ males and two (4\%) females. The majority of respondents (71\%) was between the ages of 22-30 years of age, had been flying between 5-10 years (42\%), and had logged more than 2,501 flight hours $(42 \%)$. Fifty percent of the responding pilots in training learned to fly at FAA FAR Part 141 pilot schools and the majority of pilots $(69 \%)$ had not been recently hired as a regional airline pilot.

In addition, the respondents were asked to provide information regarding their flying experience. The majority of respondents $(73 \%)$ indicated that they learned to fly on analog only instruments. Approximately one-third (35\%) of the pilots in training received no digital training/TAA training during their initial instruction. Again, about one-third (35\%) of the pilots had one - two years of glass cockpit experience. Lastly, 44 percent of responding pilots in training had logged between 50-100 hours of IMC time.

The Likert scale statement that examined the primary maneuver based (stick and rudder) training received by the pilots in training is presented in Table 1. The majority of participating pilots (83\%) reported that they had primarily received maneuver based (stick and rudder) training. Only five pilots 
$(10 \%)$ did not receive maneuver based (stick and rudder) training as their primary training method.

Table 1. Primarily Received Stick \& Rudder Training

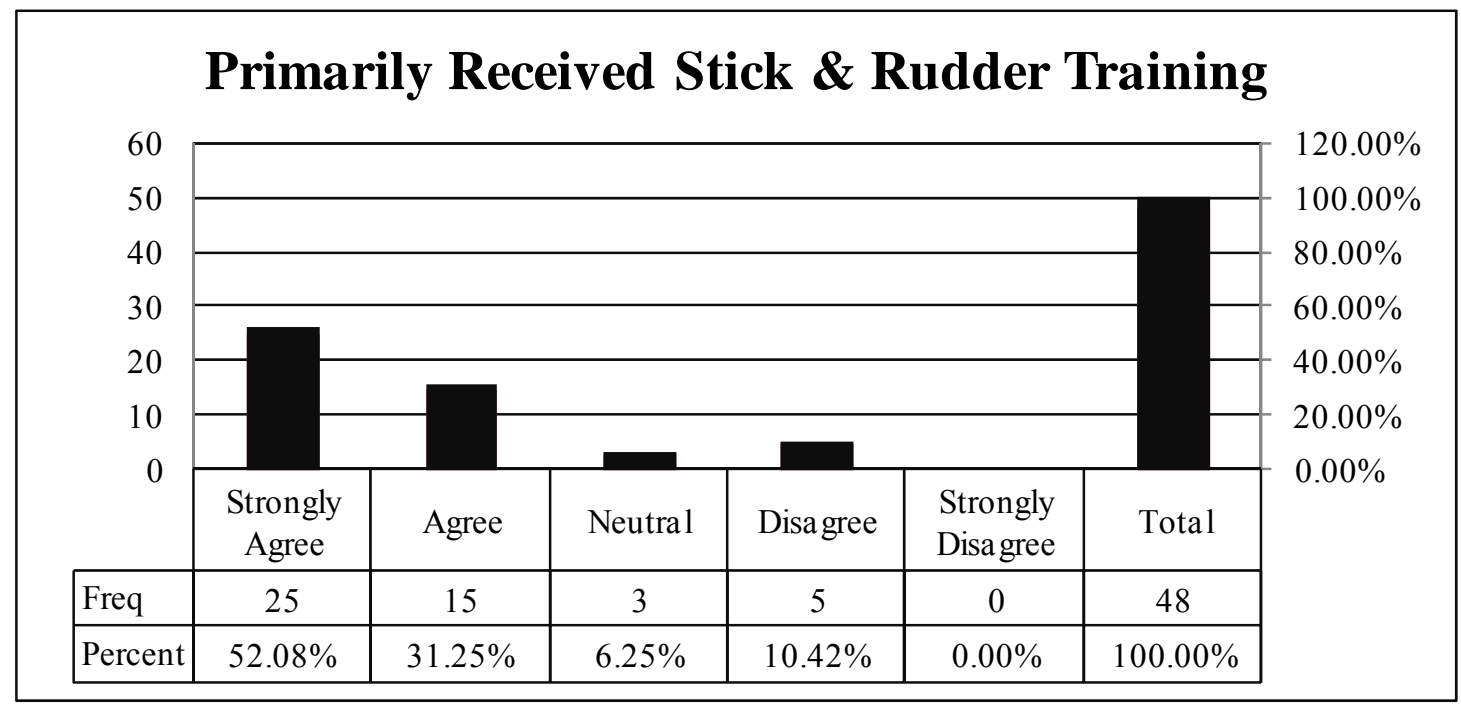

Eighteen pilots $(38 \%)$ reported that they had primarily received scenario based FITS training as indicated in Table 2. Fourteen pilots $(29 \%)$ did not receive scenario based FITS as a primary training method. There were fifteen pilots $(31 \%)$ that provided a neutral response to this question. Pilots cannot primarily receive both maneuver based (stick and rudder) training and primarily receive scenario based FITS training. While a pre-test of the questionnaire was conducted with professional pilots, the responses to this question may indicate that some pilots in training did not understand the question, or that some did not fully understand scenario based FITS training.

Table 2. Primarily Received FITS Training

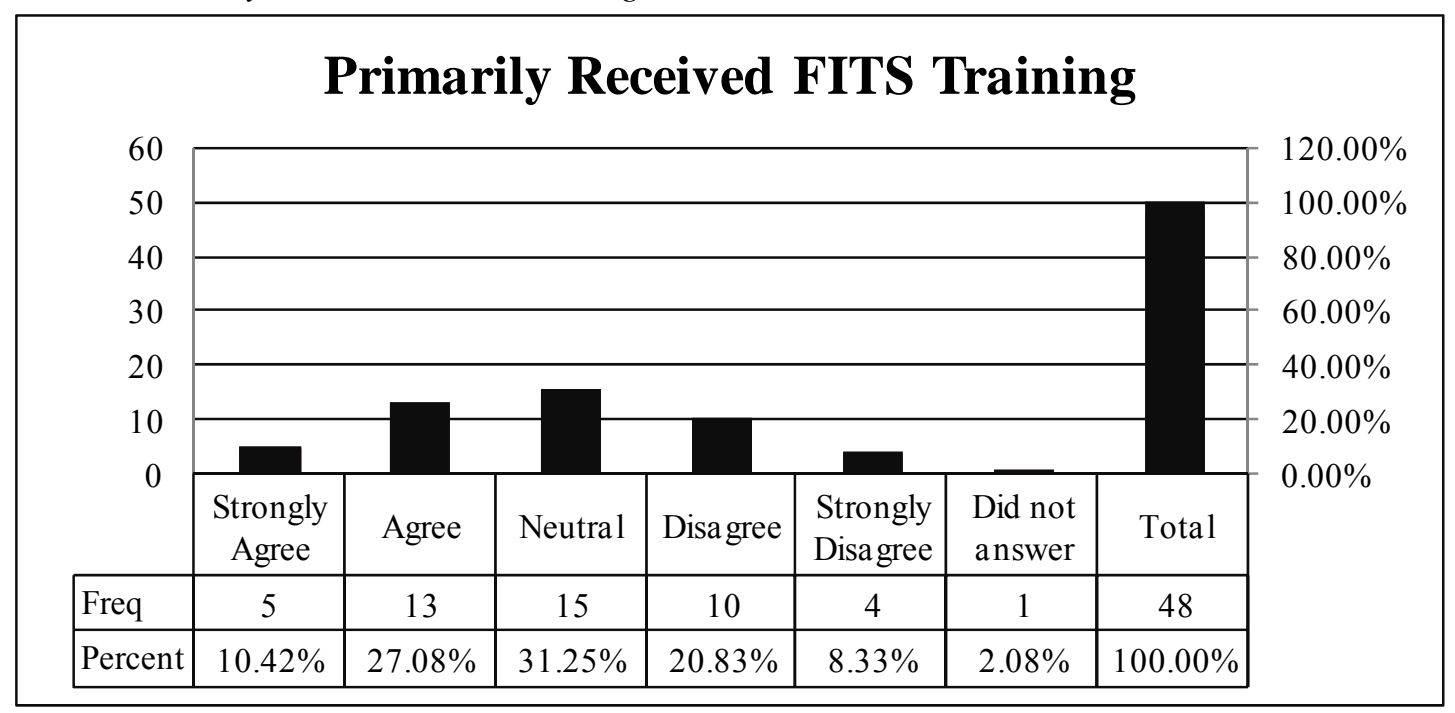


Seventeen pilots (35\%) responding to this study reported that their initial flight training had prepared them for flying TAA. An equal number of pilots disagreed their initial flight training prepared them for TAA. The remaining pilots $(29 \%)$ provided a neutral response to this question. Interestingly, despite the fact that no statistical significance was found between the research groups, pilots in this study seemed to be split about whether or not their training prepared them to fly TAA - regardless of type of training.

As shown in Table 3, only two pilots (4\%) agreed they still prefer to fly analog instruments after completing transition training. The majority of pilots $(62 \%)$ stated they did not prefer to fly analog instruments after completing transition training. Surprisingly, seventeen pilots $(35 \%)$ provided a neutral response to this question.

Table 3. Prefer Flying Analog Instruments

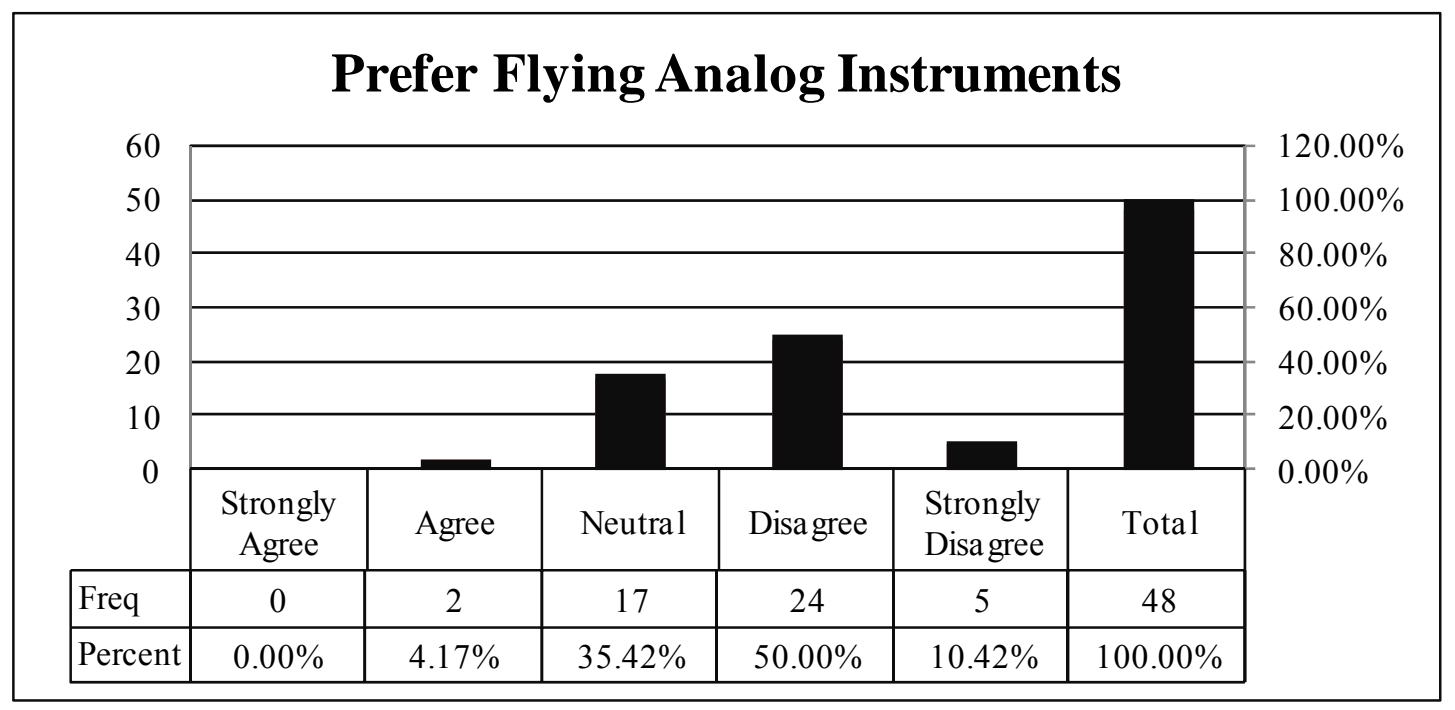

Table 4 indicates that the majority of pilots $(67 \%)$ agreed that a combination of analog and digital training made it easier to transition to technically advanced cockpits. Only four pilots $(8 \%)$ disagreed that a combination of analog and digital training made it easier to transition to technically advanced cockpits.

Regarding another question, seven pilots (14\%) agreed that there is no significant difference between analog and digital training when transitioning to technically advanced cockpits. However, the majority of responding pilots $(58 \%)$ disagreed that there is no significant difference between analog and digital training when transitioning to advanced technology cockpits. 
Table 4. Combination Analog and Digital Training Makes Easier Transition

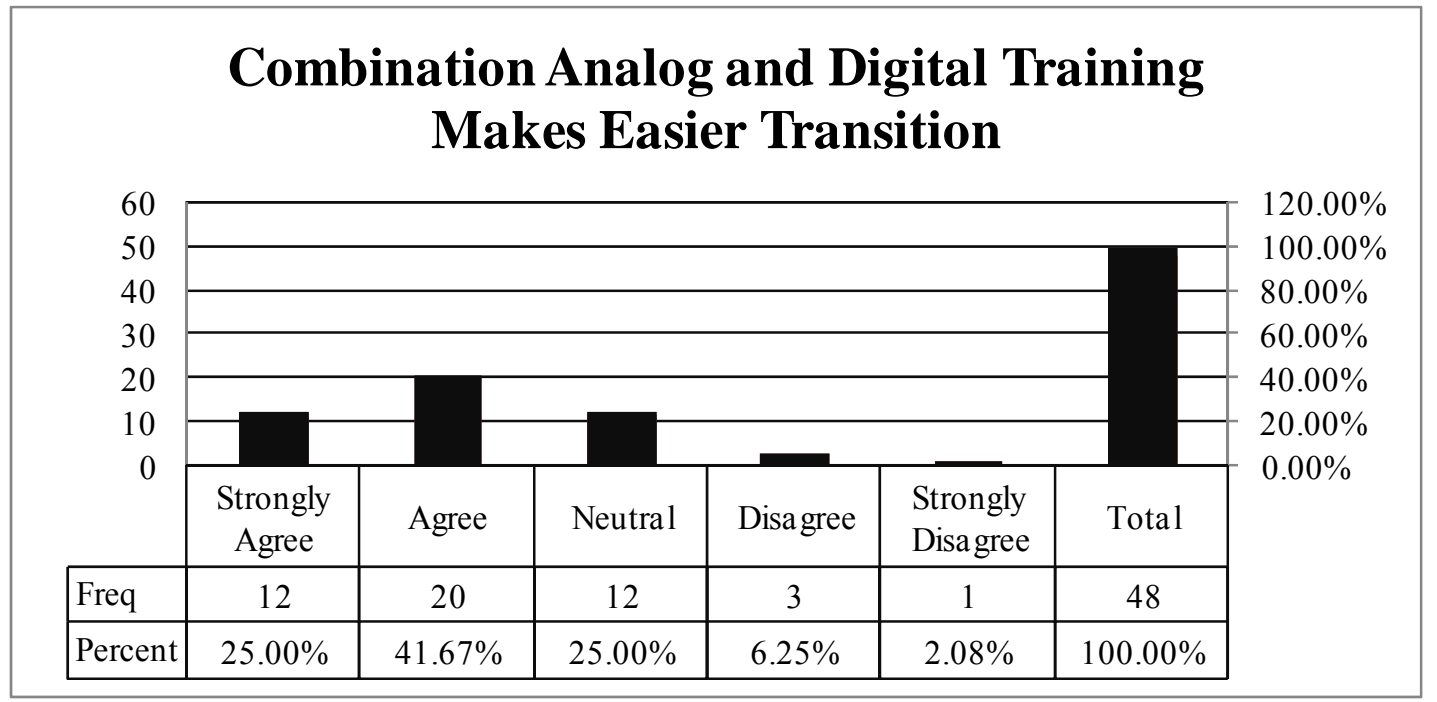

Forty-one pilots (85\%) agreed that using advanced technology made a safer pilot. Only one pilot (2\%) disagreed that using advanced technology made a safer pilot. In a follow-up question (Table 5), fifteen pilots (31\%) agreed that using advanced technology weakens basic piloting (stick and rudder) skills. However, seventeen of the pilots (35\%) disagreed that using advanced technology weakens basic piloting (stick and rudder) skills. Once again the results for this question are particularly interesting. The pilots surveyed are nearly evenly split on this question.

Table 5. Using TAA Weakens Stick and Rudder Skills

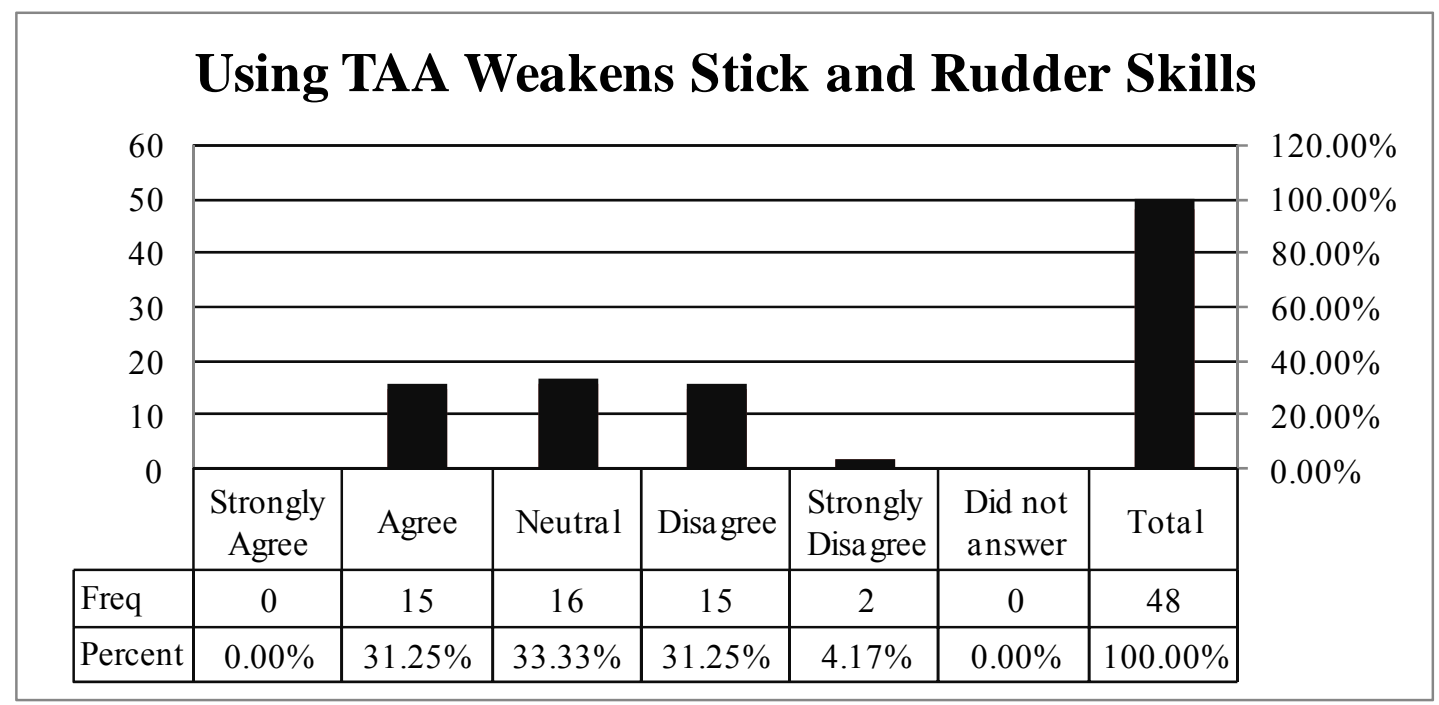

To examine the difference on the 22 Likert scale questions across the two remaining pilot groups (analog vs. analog/digital), an analysis of variance (ANOVA) was conducted. This analysis determined 
that the model was not significant, Wilkes' $\lambda=0.25, F(44,40)=0.90, p=0.63$, Partial $\eta^{2}$ (effect size) $=$ 0.50 , Power $=0.67$, indicating that no significant difference exists on the 22 Likert scale questions by the analog vs. analog/digital groups. A low Wilkes score of 0.25 indicates a departure from a normal distribution for this population and constitutes a violation of one of three assumptions necessary for an ANOVA. As is the case with most of the individual $F$ scores, the grand mean $F$ score $(0.90)$ is low and is not significant. The p score of .063 is larger than .05 which was set as the alpha. Therefore, there is greater than a .05 or $5 \%$ chance that an error will occur and cause a Type I error (i.e. rejecting the null hypothesis when it is actually true). Effect size was .50 and is a measure of the quality of the strength of the difference between the two variables and is used to determine whether the difference between the groups is meaningful in a practical sense. An effect size of .50 is considered medium. The greater the effect size, the more meaningful is the statistical difference identified by the $F$ score. Since most of the $F$ scores were not statistically significant, the effect size is irrelevant. Lastly, the Power was 0.67. Power was set at .80 for this investigation. Setting the power at .80 means there is a $20 \%$ chance of committing a Type II error. A Type II error occurs when the researcher rejects the alternative hypothesis (i.e. fails to reject the null hypothesis) when the alternative hypothesis is actually true. The higher the power, the less likely a Type II error will occur. A power of 0.67 means there is a $33 \%$ chance of committing a Type II error. Since most of the $F$ scores were not statistically significant, the power is irrelevant as well.

The qualitative component was administered using an open-ended opinion survey of IP to determine their opinions regarding the ability of a newly trained regional airline pilot's ability to learn and use advanced technology. Five specific questions were presented to the IP. These questions and the responses from the IP are stated:

1. Do you believe that scenario-based training improves the ability of a pilot/crew to master TAA? Why or why not?

- IP 1: No. I believe that basic aviation skills should be achieved first. A student should be able to fly aircraft in all modes (auto, semi auto and manual) before attempting scenario based training. Until the student feels comfortable with all Glass Cockpit and scenario training.

- IP 2: I believe scenario based training improves pilots' abilities . . period. I don't think it makes you any better for an antique DC-9 or a CRJ.

- IP 3: Yes it does improve crew's ability in advanced aircraft in that there is a relationship to realistic type flying one can expect.

- IP 4: Yes, a scenario based training event encourages the student to consider and deal with multiple variables occurring in real time as opposed to a single profile/emergency etc. . . It is especially valuable for upgrade candidates.

The majority of the IP (75\%) believed that scenario based training does improve the PT ability to master TAA. One IP (25\%), however, thought that pilots must first master maneuver based (stick and rudder skills) training before attempting scenario based training in TAA.

2. What is your opinion regarding the impact of TAA on the ability of pilots/crews to maintain stick and rudder skills?

- IP 1: Because the pilots rely so much on and use the automation, basic aviation skills suffer. Additional periodic simulator refreshers would help overcome this.

- IP 2: Flying a glass cockpit aircraft diminishes the stick and rudder skills of that used for general 
aviation.

- IP 3: Advanced aircraft such as a CL-65, a pilot becomes a manager of automation and computers. It takes away stick and rudder skills unless the pilot flies aircraft without the autopilot.

- IP 4: Students have a tendency to rely on automation too much and often, after a problem, they attempt to change automation settings when they should just fly.

All of the IP (100\%) agreed that the use of TAA and automation creates new challenges with regards to maintaining basic stick and rudder skills. Most believed that pilots who fly TAA will require additional simulator training and/or must periodically "hand fly" the airplane to maintain basic maneuver (stick and rudder) skills.

3. Do you believe that the type of initial flight training has an impact on the ability of new regional airline pilot transition to TAA? If so, what in your opinion is the best mix/type of initial training?

- IP 2: I have seen the best performance come from pilots who learned to fly gliders first.

- IP 3: Yes. Having a background from a bridge program helps, nevertheless, it does not replace experience and logged aircraft time.

Only $50 \%$ of the IP answered this question. Neither IP actually addressed the question as stated. This question was apparently unclear and no inference can be made from the answers provided. However, specific reference is made to training and experience that occurs after initial flight training.

4. In your opinion, is there a significant performance difference among newly hired pilots based on their initial type of flight training? If so, which pilots perform at higher levels and why?

- IP 2: The low time pilots that go to a regional jet specific training course like Jet U. or Simuflite are far below a pilot who has flown the King Air for 500 hours.

- IP 4: Bridge program students seem more technically proficient, but have more trouble with the big picture and decision making - CFI, freight dogs, etc . . . have trouble with the level of automation and standardization.

Only $50 \%$ of IP answered this question. Again, neither IP actually addressed the question as stated. However, these IP both agreed that experience (usually measured in flight hours, by type of aircraft, and by type of flight experience) is a relevant factor when transitioning to TAA.

5. In your opinion are none, some, or all of your pilots in training completing training at Pinnacle Airlines completely comfortable flying advanced technology aircraft? Why or why not?

- IP 1: Not all pilots are completely comfortable. Those with computer skills appear to be most comfortable. Those who have had previous experience as part of a crew using advanced technology are most comfortable.

- IP 2: About 25\% wash out that I have seen. The "career change at 50 years of age" and the "rich kid spoiled brat 18-21 years old" are the two worst types. Both are at opposite ends of the spectrum. One can't get it because they are too old and the other expects it to be handed to them because that's how life has been since birth. Both types are frustrating to work with. Little progress/success is usually made. 
Only $50 \%$ of IP answered this question. Specific reference is made to level of computer/automation proficiency, experience as crew members and age as relevant factors when transitioning to TAA.

Qualitative analysis of the data revealed two themes about IP perception of PT ability to transition to TAA. One theme centered on the role of scenario based training during initial flight training. The second theme was that other factors may play a larger role in adaptation to TAA then type of training (i.e. analog verses digital instrumentation training). Other variables may include: the total number of logged flying hours, pilot age, type of aircraft flown, experience as members of flight crews, the quality and efficiency of bridge programs, and automation skills. Consequently, a major hypothesis generated from the qualitative analysis was that maturation (the natural changes of the participants over time) and/or specific experience maybe more relevant to success for PT when transitioning to TAA.

\section{CONCLUSION}

Most of the newly trained regional airline pilots (69\%) participating in this research study agreed that the type of initial instrument training was significant when transitioning to TAA. However, no statistical evidence from this investigation was found to support this opinion. Additionally, PT were evenly divided in their belief that their initial flight training had sufficiently prepared them for transition to TAA. The obvious conclusion is that a closer examination of initial flight training is warranted. Even if initial flight training was sufficient, as would appear to be the case given the pilot response to ease of transition, many of the responding pilots perceived it not to be the case; so then, what changes to initial flight training might change the perception of these pilots in training?

Despite the fact that three groups of PT were initially surveyed in this investigation, the groups were of significantly unequal size and the vast majority received analog only training. Perhaps all of these pilots, as a whole, were much more similar than they were different. Most of these pilots learned to fly on analog instrumentation. While the IP were not asked about their initial training, it is likely they learned to fly on analog instrumentation too. Therefore, it may be concluded that this study was conducted prematurely. Perhaps, there are not yet enough pilots receiving initial instruction in TAA to make this comparison between pilot groups.

Even though a review of the literature regarding human factors research (Endsley \& Kiris, 1995; Lani \& Wickens, 2007; Miller \& Parasuraman, 2007) indicated numerous problems with the use and design of automation in complex commercial aircraft, only a minority of pilots in this study reported difficulty understanding the status of automation or mode display in advanced cockpits. Since all of these students were transitioning to TAA, it is possible they did not possess enough experience to make a judgment about mode display or systems state. Additionally, instructor comments seemed to indicate that their PT may experience problems when programming systems during flight. This appears to be an indication that this group of PT may not fully appreciate the complexity of the technology. Indeed, as is the case in most aviation training courses, completion of this training (including the check ride) may constitute only the beginning of the learning process rather than mastery of the subject matter.

Research cited in this investigation (Dornan, Craig, Gossett, \& Beckman, 2006; French, Blickensderfer, Ayers, \& Connolly, 2005; Robertson, Petros, Schumacher, McHorse \& Ulrich, 2006) demonstrated the benefits of scenario-based training for some aspects, but not all aspects, of learning to fly TAA. The majority of pilots in training reported that they had primarily received maneuver based 
(stick and rudder) training, but some of these pilots reported they had also primarily received scenario based FITS training. Clearly, the pilots that reported receiving both types of training did not understand the question or they did not understand the difference between the two training methods. Given that the majority of responding pilots in training indicated they first learned instruments on only analog instruments, and given that scenario based FITS training was initially designed to instruct students in TAA, these pilots may not clearly understand the differences between each method of pilot training. The majority of student pilots in this study may have had their first scenario based flight training experience in the Pinnacle Airline training program and may not fully appreciate the industry training standards component of FITS. If the majority of pilots in training received maneuver based (stick and rudder) training, they may not have had a frame of reference to make this distinction. To be absolutely fair; however, it is important to note that scenario based FITS training does not eliminate the requirement to master the maneuvers to the Practical Test Standard (PTS).

Finally, there is at least one more significant conclusion to be drawn from the responses of this group of airline pilots. The majority of the responding PT believed that using TAA makes them safer pilots; however, only 40 percent agreed that using TAA made them a better pilot. Clearly, this group of pilots was considerably less certain that TAA made them better pilots. Additionally, as a group, their responses were almost evenly divided when asked if TAA weakens stick and rudder skills. When asked if TAA makes pilots dependent on advanced systems, more pilots disagreed than agreed, but once again the pilots were divided in their opinion. A significant number (31\%) of the pilots in training agreed that TAA did cause dependence on advanced technology and 25 percent were uncertain (neutral) in their response to the question. Apparently, significant concern exists regarding the effects of TAA on basic flying skills. This perception was also noted in the IP survey results. A concluding fact of this study is the legitimate concern, according to the PT, that TAA does weaken stick and rudder skills and to some degree may cause dependence on advanced systems.

The qualitative component of this investigation provided credibility to the concept that scenario based training may be a significant factor leading to success when transitioning to TAA. Additionally, other factors besides type of initial training may be more directly linked to success or failure when transitioning to TAA.

Two themes emerged from the qualitative analysis. The first theme is that while type of training may not be a valid indicator of success when transitioning to TAA, method of training (i.e. the early introduction of scenario based training) may be a relevant factor. The majority of IP surveyed (75\%) agreed that method of flight training did make a difference. One instructor pilot stated: "I believe scenario based training improves pilots' abilities . . period." This unequivocal statement indicates that when PT approach flight training with an event driven perspective, they more easily adapt to the complex tasks associated with flying TAA as a member of a flight crew. In the words of another instructor, "scenario based training events encourage the student to consider and deal with multiple variables occurring in real time as opposed to a single profile/emergency etc. ..."

The second theme uncovered by qualitative analysis is that other factors may be more relevant to success such as: total number of logged flying hours, pilot age, type of aircraft flown, flight crew experience, the quality of bridge programs, and automation skills. A major hypothesis generated from the qualitative analysis was that maturation and/or specific experience maybe more relevant to pilots in training when transitioning to TAA. Several IP statements, previously mentioned, seem to confirm this conclusion: 
- "The low time pilots that go to a regional jet specific training course like Jet U. or Simuflite are far below a pilot who has flown the King Air for 500 hours."

- "Yes. Having a background from a bridge program helps, nevertheless, it does not replace experience and logged aircraft time."

- "Not all pilots are completely comfortable. Those with computer skills appear to be most comfortable. Those who have had previous experience as part of a crew using advanced technology are most comfortable."

In some cases these statements may confirm long standing traditional measure of success (i.e. flight hours logged), but in other cases non-aviation specific experiences, such as exposure to automation, also may be related to success.

Additional research is needed to determine how best to train future commercial airline pilots. This particular research study raised many more questions than it answered. Examples of such questions include:

- What are the effects of TAA training on safety and the retention of basic piloting skills?

- What are the effects of age and life experience when transitioning to TAA?

- What level of computer literacy is required for successful transition to TAA?

- What technology design and particularly what human-machine interface is best suited when transitioning to TAA?

- Do pilots always recognize their ability to determine mode display and/or systems status or are these skills that develop only with exposure and experience?

- How should pilot training methodology evolve as TAA become more widely used during initial training?

Perhaps it is too early to determine how best to conduct initial training of pilots in TAA. And for some student pilots, TAA are not yet an option at the flight school where they are undergoing initial training. However, the numbers of TAA are steadily increasing in general aviation and at collegiate flight schools. As is the case with the FITS literature, this type of investigation should continue. 


\section{REFERENCES}

Bilstein, R. E. (2001). Flight in America. Baltimore, MD: The John Hopkins University Press.

Dornan, W. A., Craig, P., Gossett, S., \& Beckman, W. (2006). Best evidence for the FAA industry training standard (FITS) program for pilot training in technically advanced aircraft. Symposium conducted at the National Aircraft Training Symposium, Daytona Beach Florida.

Endsley M. R. \& Kiris E.O. (1995). The Out-of-the-loop performance problem and level of control in automation. Human Factors, Vol. 37, 381-394.

FAA Education and Research (2006). Industry training standards. Retrieved October 9, 2007 from http://www.faa.gov/education_research/training/fits/

Fraenkel, J.R., \& Wallen, N.E., (2003). How to design and evaluate research in education (5 ${ }^{\text {th }}$ ed.). New York, NY: The McGraw-Hill Companies, Inc.

French, J., Blickensderfer, B, Ayers, F., \& Connolly, T. (2005). FITS combined task $1 \& 2$ final report. Final Report prepared for On Site FITS Director, Embry Riddle University, Daytona Beach, FL.

Lani. C. \& Wickens, C.D. (2007). Factors affecting task management in aviation. University of Illinois at Urbana-Champagne. Human Factors, Vol. 49, (No. 1), February 2007, 16-24.

Levene, H. (1960). In contributions to probability and statistics: Essays in honor of Harold Hotelling, I. Olkin et al. eds., Stanford University Press, 278-292.

Miller, C.A \& Parasuraman, R. (2007). Design for flexible interaction between humans and automation: Delegation interfaces for supervisory control. Human Factors, Vol. 49, February 2007, (No 1), 57-75.

Robertson, C. L., Petros, T.V., Schumacher, P. M., McHorse, C.A. and Ulrich, J.M. (2006). Evaluating the effects of FITS training. Retrieved February, 2009, from: http://www.faa.gov/education_research/training/fits/research/media/und.pdf

Shapiro, S. S. \& Wilk, M. B. (1965). "An analysis of variance test for normality (complete samples)", Biometrika, 52, (3 and 4), 591-611. 